\title{
Identification of the vibrational marker of tyrosine cation radical using ultrafast transient infrared spectroscopy of flavoprotein systems
}

\author{
Katalin Pirisi ${ }^{1}$ - Lipsa $\mathrm{Nag}^{2} \cdot$ Zsuzsanna Fekete $^{1}$. James N. Iuliano ${ }^{3}$. Jinnette Tolentino Collado ${ }^{3} \cdot$ lan P. Clark ${ }^{4}$. \\ Ildikó Pécsi ${ }^{1}$. Pierre Sournia ${ }^{2}$. Ursula Liebl ${ }^{2}$. Gregory M. Greetham ${ }^{4} \cdot$ Peter J. Tonge ${ }^{3} \cdot$ Stephen R. Meech $^{5}$. \\ Marten H. Vos ${ }^{2} \cdot$ Andras Lukacs ${ }^{1}$
}

Received: 11 January 2021 / Accepted: 9 February 2021

(c) The Author(s) 2021

\begin{abstract}
Tryptophan and tyrosine radical intermediates play crucial roles in many biological charge transfer processes. Particularly in flavoprotein photochemistry, short-lived reaction intermediates can be studied by the complementary techniques of ultrafast visible and infrared spectroscopy. The spectral properties of tryptophan radical are well established, and the formation of neutral tyrosine radicals has been observed in many biological processes. However, only recently, the formation of a cation tyrosine radical was observed by transient visible spectroscopy in a few systems. Here, we assigned the infrared vibrational markers of the cationic and neutral tyrosine radical at 1483 and $1502 \mathrm{~cm}^{-1}$ (in deuterated buffer), respectively, in a variant of the bacterial methyl transferase TrmFO, and in the native glucose oxidase. In addition, we studied a mutant of AppABLUF blue-light sensor domain from Rhodobacter sphaeroides in which only a direct formation of the neutral radical was observed. Our studies highlight the exquisite sensitivity of transient infrared spectroscopy to low concentrations of specific radicals.
\end{abstract}

\section{Introduction}

Charge transfer processes are omnipresent in biological reaction pathways. Alongside specialized redox-active cofactors, in many cases amino acid residues—mostly aromatic

Pushing the limits of flash photolysis to unravel the secrets of biological electron and proton transfer - a topical issue in honour of Klaus Brettel.

Marten H. Vos

marten.vos@polytechnique.edu

$\triangle$ Andras Lukacs

andras.lukacs@aok.pte.hu

1 Department of Biophysics, Medical School, University of Pecs, Szigeti Str. 12, 7624 Pecs, Hungary

2 Laboratoire d'Optique et Biosciences, CNRS, INSERM, Ecole Polytechnique, Institut Polytechnique de Paris, 91128 Palaiseau, France

3 Department of Chemistry, Stony Brook University, Stony Brook, NY 11794-3400, USA

4 Central Laser Facility, Research Complex at Harwell, Harwell Science and Innovation Campus, Didcot OX11 0QX, Oxon, UK

5 School of Chemistry, University of East Anglia, Norwich NR4 7TJ, UK residues like tryptophan and tyrosine-play important roles in reaction intermediates. Therefore, cationic and neutral radicals are thought to be important. They can be investigated using spectroscopic methods, including EPR, visible and infrared spectroscopy. The latter two can be combined with very high time resolution, allowing the characterization of very short-lived intermediates in photo-activatable systems. A prototypical example is the formation of tryptophan cation radicals during photoactivation of the flavophotoenzyme DNA photolyase where sequential electron transfer involving three adjacent tryptophans occurs on the picosecond timescale [1-5]. The pKa of the tryptophan cation radical is $\sim 4$ and its deprotonation happens in a couple of hundreds of nanoseconds [2]. This process, and similar subsequently studied processes in the related cryptochrome blue-light sensors [6-11] have been extensively studied by polarization and spectrally resolved transient visible spectroscopy. Transient infrared spectroscopy was successfully used to characterize protein dynamics, and activation mechanism of light dependent protein function [11-14]. The sensitivity of time-resolved infrared method allows us to visualize in the difference infrared spectra the vibrational modes of the chromophore or protein arising upon photoexcitation. Previously, we employed transient infrared spectroscopy to characterize the photocycle of the BLUF photoreceptor 
PixD, and we showed that the photoactivation mechanism of PixD involves a sequential formation of the anionic and neutral flavin radicals upon photoexcitation of the flavin chromophore, and the conserve tyrosine is the source of both electron and proton in the PCET mechanism [15].

Tyrosine cation radicals may also play a role as reaction intermediates, but they are thought to be far less stable and more short-lived, or only occurring concomitant with proton transfer, as its pKa is - 2 [16]. The deprotonated, neutral, tyrosine radical has been observed as a relatively long-lived functional intermediate in a number of proteins, including photosystem II and type II ribonucleotide reductase [17, 18], and its visible [19] and vibrational [20] spectroscopic characteristics have been determined. Yet, only recently Vos and co-workers used transient visible spectroscopy to identify a short-lived protonated cation radical after photoexcitation of flavin in non-photoactive flavoenzymes where tyrosine and the isoalloxazine ring systems closely interact: in a variant of the bacterial flavoenzyme TrmFO [21], a tRNA: ${ }^{5} \mathrm{U} 54-$ methylase, and subsequently in native glucose oxidase [22].

The available crystal structure [23] of the purified TrmFO shows a close cysteine at position 51 (C51) in the Thermus thermophilus enzyme, which forms a stable adduct with the flavin, a property mainly observed in LOV domains [24]. The adduct formation in LOV proteins is triggered by light absorption of FMN and a nearby conserved cysteine forms an adduct with the $\mathrm{C} 4 \mathrm{a}$ atom of the flavin. Mutation to the conserved cysteine residue blocks the formation of the adduct in LOV domains, resulting in a highly fluorescent protein. Contrary to the observation in LOV domains, the adduct formed in TrmFO is highly fluorescent. Substitution of C51 with alanine inhibits the adduct formation, yielding an almost non-fluorescent flavin since a tyrosine residue (Y343) is very close and parallel to the isoalloxazine ring (Fig. 1); this is a favorable position of Y343 for making the electron transfer process very efficient and the excited state of the flavin very short-lived, $\sim 1$ ps or less. In the same transient visible studies [21], Vos and co-workers assigned a photoproduct to the $\mathrm{FAD}^{-\mathrm{T}} \mathrm{TyrOH}^{++}$state, that subsequently decayed in $\sim 3$ ps.

TrmFO system appears to be a good model system to investigate infrared properties of a tyrosine cation radical. Therefore, we performed transient infrared measurements both on TrmFO C51A and the C51A/Y343F double mutant. The substitution of tyrosine with phenylalanine was chosen to inhibit the electron transfer pathway. The results suggest the presence of parallel reaction schemes and the sensitivity of infrared spectroscopy to observe low concentrations of reaction intermediates. In addition, we expand our studies to glucose oxidase and BLUF proteins to further validate the presence of tyrosine cation radical in those systems.

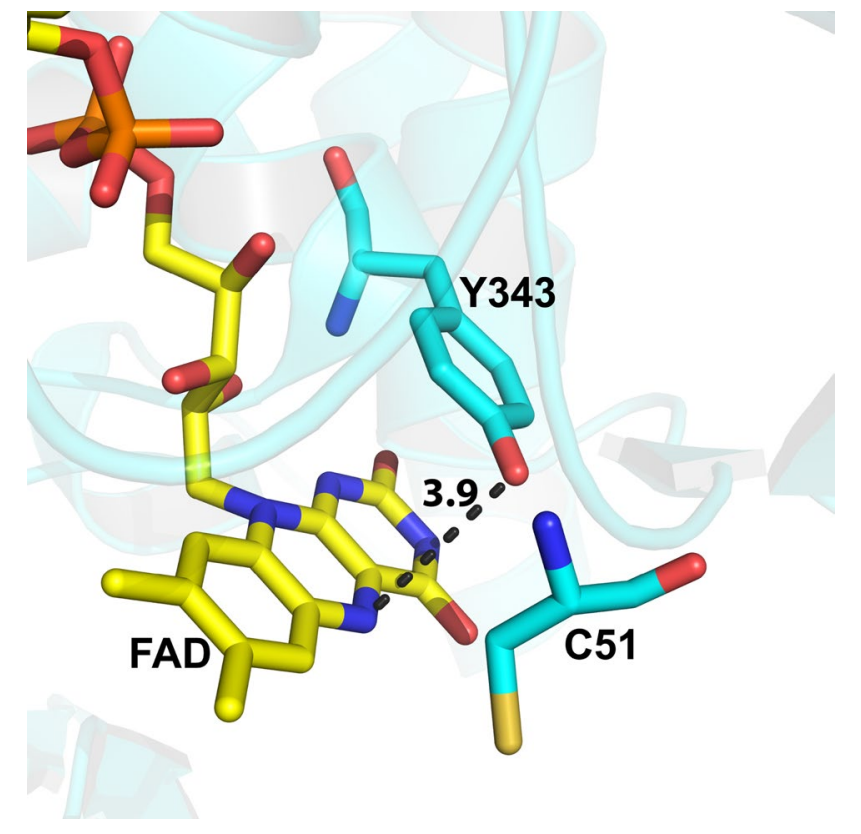

Fig. 1 Crystal structure of Thermus thermophilus TrmFO (pdb 3G5Q). In this study, the residue C51 was mutated to A creating the C51A TrmFO mutant

\section{Experimental methods}

\subsection{Transient infrared absorption measurements}

Ultrafast time-resolved IR (TRIR) spectra were measured at the STFC Central Laser Facility with 100 fs time resolution [25]. TRIR spectra were acquired at $20{ }^{\circ} \mathrm{C}$ from 1300 to $1800 \mathrm{~cm}^{-1}$ at a resolution of $3 \mathrm{~cm}^{-1}$ per pixel. Data were obtained using a $50 \mu \mathrm{m}$ path length cell, while the sample was rastered with the help of a Lissajous scanner to minimize long-lived phototransformation (photobleaching, photodegradation and photoconversion). The excitation beam of the $450 \mathrm{~nm}, 100 \mathrm{fs}, 5 \mathrm{kHz}$, pulses was focused to a spot size of $\sim 100 \mu \mathrm{m}$ and the pulse energy was kept around $500 \mathrm{~nJ}$. Transient difference spectra (pump on-pump off) were recorded using the IR probe at time delays between $1 \mathrm{ps}$ and $2 \mathrm{~ns}$. After the measurements were recorded, the extent of phototransformation was shown to be negligible using absorbance spectroscopy. Spectra were calibrated relative to the IR transmission of a pure cis stilbene standard sample placed at the sample position. TRIR of lightadapted W104Y AppA BLUF domain were recorded under continuous illumination with $\sim 200 \mathrm{~mW}$ of $450 \mathrm{~nm}$ light on the sample cell during the experiment.

Data were analyzed globally using the Glotaran software package [26]. In the case of global analysis, simultaneous fit is performed on the measured $\Delta \mathrm{A}$ data, the pre-exponential factors (belonging to the different 
wavelengths) of each decay rate will give the decay-associated spectra (DAS). An alternative way is to assume a sequential model $(\mathrm{A} \rightarrow \mathrm{B} \rightarrow \mathrm{C})$ and solve the respective differential equations. The obtained evolution-associated spectra (EAS) will show the evolution of spectra in time [27]. All the data presented in the paper were fitted assuming a sequential scheme, the decay rates for the individual EAS spectra show the time needed to convert from one state to the other. Assuming the simplest $\mathrm{A} \rightarrow \mathrm{B} \rightarrow \mathrm{C}$ scheme, the decay rate of EAS1 is the time need to evolve from state A to state B, the spectrum of EAS1 is the spectrum belonging to state $\mathrm{A}$.

Individual kinetics were fit using Origin 2020 from Originlab.

\subsection{Protein expression and purification}

The BLUF domain of AppA (AppA $\mathrm{BLUF}_{\text {: }}$ residues 5-125) was expressed in BL21(DE3) Escherichia coli cells. Protein expression and purification were performed in the dark as described previously [28]. The W104Y AppA BLUF construct was obtained by site-directed mutagenesis performed using Phusion site-directed mutagenesis kit (NEB), wt $\mathrm{App}_{\mathrm{BLUF}}$ in $\mathrm{pET} 15 \mathrm{~b}$ as a template and the following primers: 5'-CGCCGCTTTGCGGGATATCACATGCAGCTC TCC-3' and 5'-CTTGGCGATCGGTTCCTCTGCGAGGAT CTC-3'. All constructs were verified by DNA sequencing.

C51A and C51A/Y343F mutant proteins of TrmFO from T. thermophilus were heterologously expressed and purified as previously described [21]. Glucose oxidase from $A$. niger was purchased from Sigma and dissolved in the same D2O buffer (50 mM NaH2PO4, $10 \mathrm{mM} \mathrm{NaCl}, \mathrm{pD} 8.0)$ as the TrmFO and AppA W104Y mutants. All protein concentrations were kept to $\sim 1 \mathrm{mM}$ and measured in Harrick cells using $50 \mu \mathrm{m}$ spacers between the $\mathrm{CaF}_{2}$ windows; in this way, $\sim 80 \mu \mathrm{l}$ sample was used for one measurement.

\section{Results}

\subsection{TrmFO mutants C51A/Y343F and C51A}

In C51 A/Y343F double mutant of TrmFO, the fluorescence quenchers nearby the flavin are absent, and the FAD* excited state decays in a multiphasic manner on the timescale of $30 \mathrm{ps}$ and longer [21]. Accordingly, TRIR spectra of C51A/ Y343F mutant (Fig. 2a) resemble the TRIR spectra of oxidized flavin in solution (see Fig. S1) on the fs and ps timescale, and do not evolve with time, thus it can be assigned to $\mathrm{FAD}^{*}$ vibrational modes. The negative vibrational modes (bleaches) are assigned to the depletion of the ground-state population of the flavin while the positive peaks (called transients in this paper) are associated with vibrational modes of the excited state of flavin or to protein modes. The 1547 and $1581 \mathrm{~cm}^{-1}$ bleaches were assigned to flavin ring modes, the $\sim 1700 \mathrm{~cm}^{-1}$ bleach was earlier assigned as the vibration of the $\mathrm{C} 4=\mathrm{O}$ carbonyl of flavin, the $1640-1650 \mathrm{~cm}^{-1}$ bleach was assigned as the $\mathrm{C} 2=\mathrm{O}$ stretch, and the 1380 and $1415 \mathrm{~cm}^{-1}$ transients are vibrational markers of the excited state of the flavin (see assignment of these peaks in the first paragraph of SI) [12, 28-30].

The overlaid TRIR spectra of C51A/Y343F and C51A variants (Fig. 2c) illustrate that the frequency of $\mathrm{C} 4=\mathrm{O}$ in the $1700 \mathrm{~cm}^{-1}$ region is blue shifted by approximately $8 \mathrm{~cm}^{-1}$, from $1697 \mathrm{~cm}^{-1}$ in C51A mutant to $1705 \mathrm{~cm}^{-1}$ in C51A/Y343F. This suggests that the replacement of Y343 to phenylalanine decreases the hydrogen bonding around the C4 carbonyl of Flavin.

The kinetics of the ground-state recovery observed at $1547 \mathrm{~cm}^{-1}$ (Fig. 2f) highlights that the C51A mutant returns to the ground much faster (within a few ps) than the double mutant. In C51A TrmFO, there is a faster charge transport to the tyrosine, followed by charge recombination to the resting FAD state [21], whereas in the C51A/Y343F mutant, the flavin fluorescence is far less quenched on this time scale.

The TRIR spectra of TrmFO C51A mutant evolve significantly (Fig. 2b), presumably reflecting excited state decay, and charge transfer processes, which differ from those of the C51A/Y343F mutant assigned to FAD*. At lower frequencies, the C51A spectra show two broad transients around $1485-1505 \mathrm{~cm}^{-1}$ and $1515-1535 \mathrm{~cm}^{-1}$ which are not present in the $\mathrm{C} 51 \mathrm{~A} / \mathrm{Y} 343 \mathrm{~F}$ mutant. Hence, we propose that the appearance of these vibrational modes is primarily related to the electron transfer process, which takes place from tyrosine 343 to the flavin.

Based on previous infrared measurements on flavin radicals, the broad transient around $1515-1535 \mathrm{~cm}^{-1}$ can a priori be interpreted relatively straightforward. It appears composed of two shoulders-at $1518 \mathrm{~cm}^{-1}$ and $1528 \mathrm{~cm}^{-1}$ whose relative intensity varies in time, reflecting two distinct vibrational modes. In earlier work [31], we used DFT calculations and infrared measurements to assign these two transients to the formation of the anionic, FAD ${ }^{-},\left(1518 \mathrm{~cm}^{-1}\right)$ and neutral, FADH, flavin radicals $\left(1528 \mathrm{~cm}^{-1}\right)$. For example, in the case of PixD-a BLUF domain protein-the sequential formation of these two peaks shone light on the photoactivation process of the protein: first, the anionic flavin radical $\left(\mathrm{FAD}^{-}\right)$was formed within $2-3$ ps followed by the protonation of the flavin, thus leading to the formation of the neutral flavin radical (FADH') in $\sim 100 \mathrm{ps.}$

A similar sequence appears to be observed in the case of the C51A mutant. A global analysis of the TRIR data yielded time constants of 1 and $3 \mathrm{ps}$, and a very small longer lived phase (Fig. 2d), similar to the one observed by Nag et al. [21]. Using a sequential scheme to fit data adequately, the evolution-associated spectra (EAS) obtained from the 

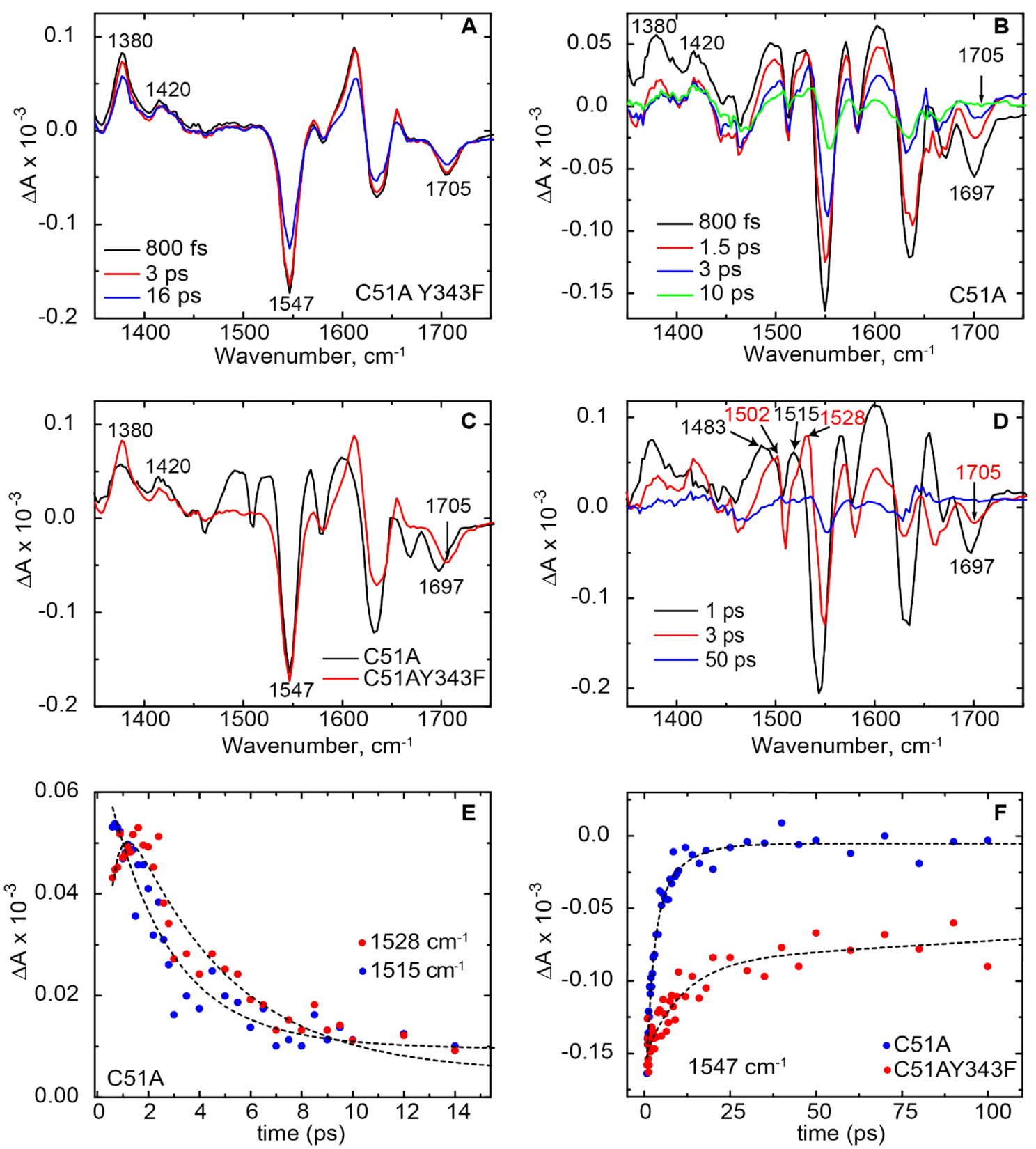

Fig. 2 a TRIR spectra measured on the C51AY343F mutant at different delays. b TRIR spectra measured on the C51A mutant at different delays. $\mathbf{c}$ Comparison of TRIR spectra measured on the C51A and C51AY343F mutants at 800 fs. d EAS spectra of the C51A mutant.

e Kinetics measured on the C51A mutant at $1515 \mathrm{~cm}^{-1}$ (blue) and $1528 \mathrm{~cm}^{-1}$ (red), respectively. f Kinetics at $1547 \mathrm{~cm}^{-1}$ in the C51A and C51AY343F mutant

global fit are well separated (Fig. 2d). EAS1 is mainly dominated by the vibrational modes of FAD*-FAD (cf. Fig S1), as expected from our previous work using transient visible spectroscopy [21]. However, we also observed a distinct transient formed at $1515 \mathrm{~cm}^{-1}$, indicating that some FAD $^{--}$is formed at very early times after excitation. This suggests that a fraction of the excited state decays, by flavin photoreduction, much faster than the time resolution of our system and the previously reported [21] fluorescence

experiments $(\sim 200 \mathrm{fs})$. Indeed, the kinetics at $1515 \mathrm{~cm}^{-1}$ rise with the instrument response time.

EAS2 (3 ps) includes a substantial transient at $1528 \mathrm{~cm}^{-1}$ which was assigned to FADH. The kinetics of $1528 \mathrm{~cm}^{-1}$ transient (Fig. 2e) shows that this signal does not form instantly after excitation but in a sequential way, in $\sim 1 \mathrm{ps}$ after the initial formation of the anionic radical. This is an unexpected result since FADH has a marked visible signature and was not observed $(<10 \%)$ in the visible transient 
absorption experiments [21, 32], where the 3-ps phase was assigned to recombination of $\mathrm{FAD}^{-}$and $\mathrm{TyrOH}^{+}$. Therefore, it appears that transient infrared method is exquisitely sensitive to even very minor amount of FADH' observed at $1528 \mathrm{~cm}^{-1}$ in the $\mathrm{C} 51 \mathrm{~A}$ TrmFO mutant. Formation of FADH implies the presence of a proton and a proton donor, this latter obviously must be the tyrosine. As the flavin is protonated as the result of the electron transfer process, one can talk about proton-coupled electron transfer process (PCET) seen in many photoactive flavoproteins (Slr1694 BLUF, PaPB, etc.)

A second difference between the EAS1 of the C51A mutant (Fig. 2d) and the expected pure excited state spectrum observed in the C51A/Y343F mutant (Fig. 2a) is the transient between 1475 and $1500 \mathrm{~cm}^{-1}$, the maximum being around $1483 \mathrm{~cm}^{-1}$. Based on the assessment that a tyrosine cation radical is formed along with FAD reduction [21], we attribute the $1483 \mathrm{~cm}^{-1}$ transient to the tyrosine cation radical associated with the fraction of $\mathrm{FAD}^{--}$formed within the instrument response (see above). In EAS2 (3-ps phase precursor), the $1483 \mathrm{~cm}^{-1}$ transient is shifted towards $1500 \mathrm{~cm}^{-1}$. We suggest that this may be due to the expected proton transfer of the tyrosine radical to the flavin anion, yielding the small amount of FADH alluded to above, thus producing some neutral tyrosine radical. This is in accord with assignment of a $1502 \mathrm{~cm}^{-1}$ mode to the TyrO $\mathrm{C}-\mathrm{O}$ stretching vibration in a ribonucleotide reductase from Bacillus cereus by Raman spectroscopy [18] and a $1504 \mathrm{~cm}^{-1}$ FTIR band in PSII from Synechocystis sp. PCC 6803 [17].

Analysis of the EAS spectra of C51A mutant illustrates that the high-frequency mode of the flavin $\left(\sim 1700 \mathrm{~cm}^{-1}\right)$ is upshifted by $\sim \mathrm{cm}^{-1}$ in EAS2 compared to EAS1 which reflects a less hydrogen bonded environment for the $\mathrm{C} 4=\mathrm{O}$ carbonyl of the flavin. It is expected since the deprotonation of the tyrosine perturbs the hydrogen bonding network around the flavin.

As mentioned above, the ground-state recovery in the C51A/Y343F double mutant is much slower than in the C51A mutant which it is expected since close by electron donor is absent. Nevertheless, the decay of unquenched flavin excited states is faster than expected, indicating the presence of another electron donor allowing electron transfer on the tens and hundreds of picoseconds timescale, presumably a Trp residue [21]. Interestingly, all the IR spectra of the C51A/Y343F protein are similar and devoid of any sign of tryptophan or flavin radical formation. This indicates that if such radicals are formed on the timescale of our experiments, they decay faster than they are formed or the yield of formation of these radicals is under the detection limit of the present instrumentation. Experiments with additional mutations are required to identify of other potential electron donors.

\subsection{Glucose oxidase}

We collected new TRIR measurements on glucose oxidase (GOX) to further characterize the infrared vibrational markers observed for tyrosine cation and neutral radical. Previously, we published the TRIR spectra of glucose oxidase in three different redox states of flavin (oxidized, neutral and anionic radical) focusing on the vibrational markers of the neutral and anionic redox state as well as on the interpretation on the longer lived product states involving tryptophan [31]. In the present study, we globally fit the data using a sequential model to obtain the EAS spectra of the measurements.

GOX contains two tyrosine residues close to the isoalloxazine ring of FAD (Fig. 3). We recently characterized the relatively complex photochemistry of glucose oxidase using transient visible absorption and fluorescence spectroscopy [22]. We found evidence for multiphasic FAD* decay leading initially to the $\mathrm{FAD}^{--} \mathrm{TyrOH}^{++}$state in $\sim 1 \mathrm{ps}$, and subsequently also to some $\mathrm{FADH}^{-} \mathrm{TyrO}^{\circ}$ in $\sim 4$ ps and to some $\mathrm{TrpH}^{+}$in $\sim 30 \mathrm{ps}$, all these electron and proton transfer steps occur in competition with recombination reactions. In this light, we reinvestigated GOX by TRIR.

The transient infrared spectra of glucose oxidase (Fig. 4a) resemble the TRIR data of C51A TrmFO mutant (Fig. 2b): at lower frequencies, we observe the transients around 1380 and $1420 \mathrm{~cm}^{-1}$ related to the excited state of the flavin, a broad transient around $1480 \mathrm{~cm}^{-1}$ and a well separated one at $1515 \mathrm{~cm}^{-1}$, and a new transient peak appearing at $1528 \mathrm{~cm}^{-1}$ at later time delays. Figure $4 \mathrm{~b}$ depicts the result

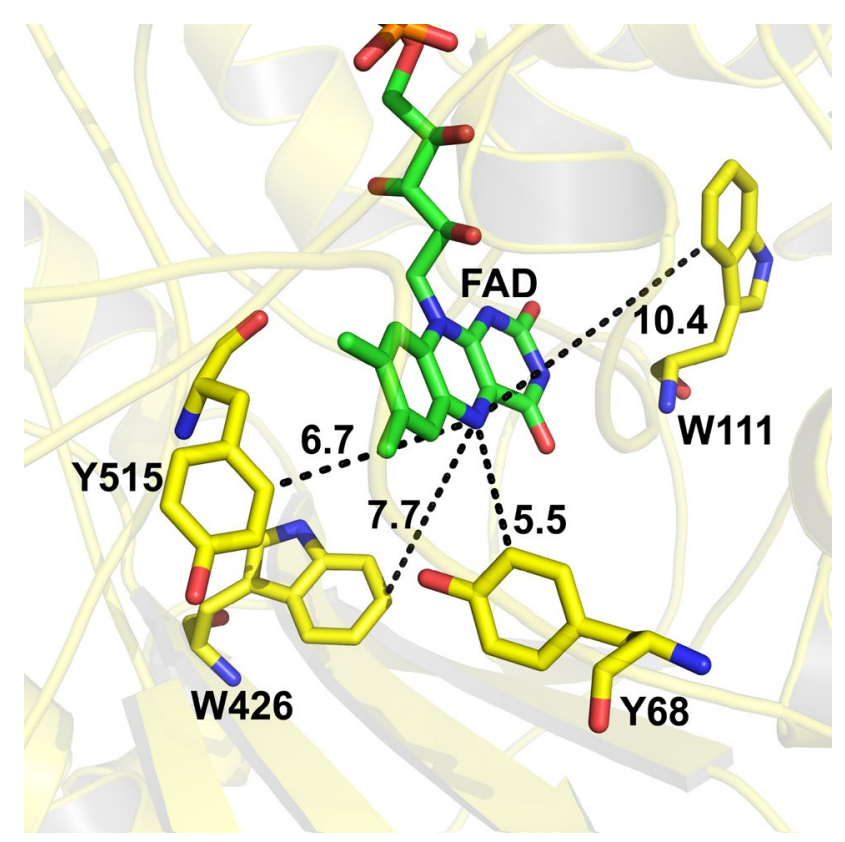

Fig. 3 Structure of glucose oxidase (PDB: 1CF3) showing the distance of isoalloxazine ring and the potential electron donors 

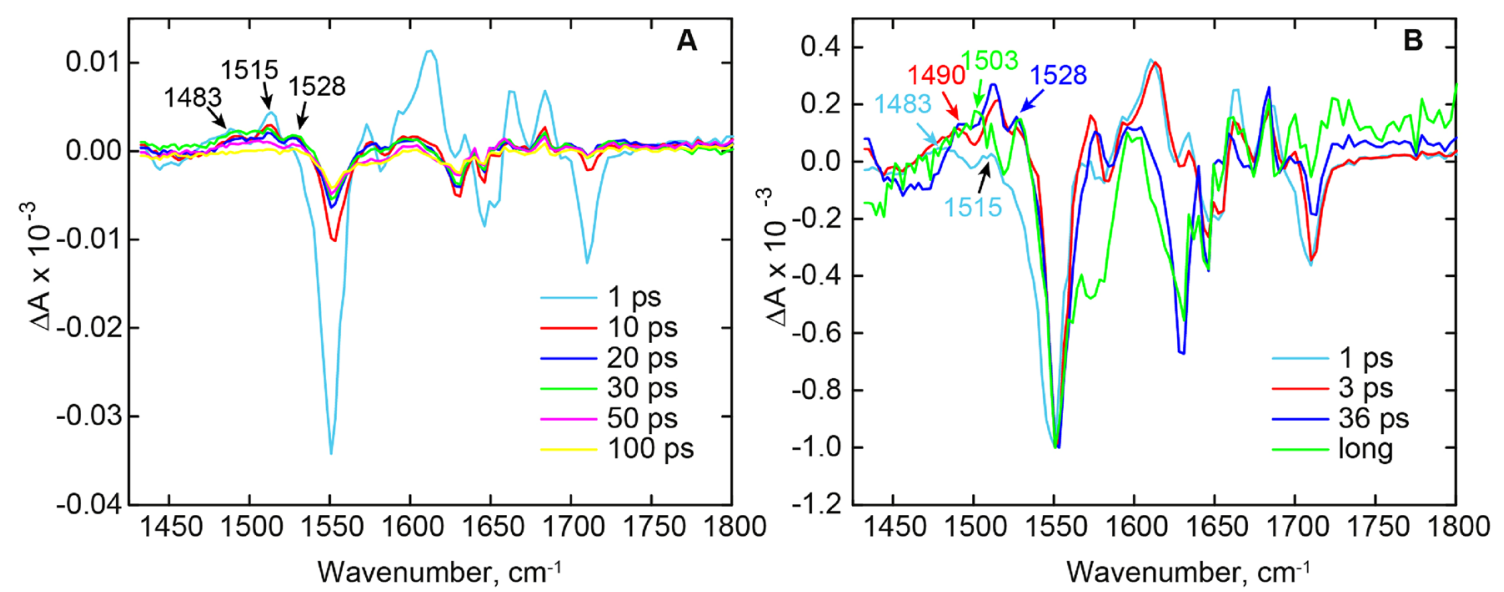

Fig. 4 TRIR on glucose oxidase. a TRIR spectra at different delays. b EAS spectra obtained from global analysis. Here, we normalized data here to highlight the vibrations

of a global fitting of TRIR dataset in terms EAS assuming a sequential model. The time constants of the fit, 1, 3, $36 \mathrm{ps}$ and the asymptotic one agree with those of the visible data, with the limitation that in the infrared measurements we could not separate the pure excited state of the flavin. EAS1 (1 ps) and EAS2 (3 ps) both contain spectral features ascribed to the excited flavin, as well as a $1483 \mathrm{~cm}^{-1}$ band and the flavin anionic peak $\left(1515 \mathrm{~cm}^{-1}\right)$. Similar to TrmFO, the tyrosine cation radical in GOX is formed in 1 ps upon excitation [22], and in the TRIR of GOX the $1483 \mathrm{~cm}^{-1}$ band appears to reflect the vibrational mode of the tyrosine cation radical, in agreement with the above assignment for C51A TrmFO.

The EAS3 and EAS4 illustrate a poorly resolved shoulder at $1490 \mathrm{~cm}^{-1}$, as well as a distinct shoulder at $1528 \mathrm{~cm}^{-1}$ which we assigned to FADH state of flavin. In our earlier work [31], we showed that electron transfer can also take place from one of the adjacent tryptophans (W426, W111), so the formation of the $1490 \mathrm{~cm}^{-1}$ band indicates the appearance of the tryptophan cation radical.

In our previous studies on AppA [30] and pixD mutants, the TRIR spectra reveal the $1488-1490 \mathrm{~cm}^{-1}$ peak arising from a photo-induced electron transfer processes, and we assigned this peak to the vibrational marker of the $\operatorname{TrpH}{ }^{+}$ radical (assignment of this peak in the PixD mutant can be found in the SI). Based on calculation, this mode is related to the $\Phi_{19 \mathrm{a}}$ mode of the phenyl ring (using the Wilson vibration nomenclature) and the C2-C3 stretch [33, 34]. Resonance Raman spectroscopy was recently utilized to measure vibrational properties of tryptophan radicals, and it was found that the $\mathrm{W} 5^{+}$vibrational mode in water can be found at $1495 \mathrm{~cm}^{-1}$, while in $\mathrm{D}_{2} \mathrm{O}$ is shifted to $1488 \mathrm{~cm}^{-1}$ [35]. Vibrational marker of tryptophan cation radical was first assigned by Blanco-Rodrigez et al. [36], using the same time-resolved transient infrared absorption system (ULTRA
[25]) what was used for this work. The authors assigned the $\mathrm{Trp}^{+}$feature at 1278 and $1497 \mathrm{~cm}^{-1}$ in the case of a model inorganic complex in DCM. This compares well (specially if we take into account the difference between the two solvents) with the expected $1490-1495 \mathrm{~cm}^{-1}$.

The long phase (EAS4) contains a (poorly resolved) transient at $1502 \mathrm{~cm}^{-1}$. Based on our observation of the visible transient data [22], where the tyrosine neutral radical is stabilized for tens of picoseconds and longer, we conclude that the $1502 \mathrm{~cm}^{-1}$ band is related to the formation of the TyrO. In the visible transient data, tyrosine radical is already formed in the EAS3 (37 ps) phase, which is probably the case in the TRIR measurements, just the $1502 \mathrm{~cm}^{-1}$ transient is covered by the small bleach of $\mathrm{FAD} \sim 1503 \mathrm{~cm}^{-1}$.

Taken all data together, the infrared absorption data provide extra details of distinct vibrational modes for neutral and cation tyrosine radical along with flavin radicals and appear generally consistent with the visible transient absorption data.

\subsection{W104Y AppA mutant}

AppA regulates the biosynthesis of the photosynthetic genes in the bacterium Rhodobacter sphaeroides. In the dark and at low oxygen level, AppA binds PpsR, a transcriptional antirepressor, while at high light intensity or oxygen level the AppA-PpsR complex dissociates, and PpsR blocks the production of the photosynthetic genes [37, 38]. The primary photochemistry of AppA is driven by the reorganization of the hydrogen bonding network around the flavin upon blue-light absorption, this small reorganization leads to a larger structural change in the protein [26]. Even though AppA is a photoactive flavoprotein, its function is not driven by electron transfer as flavin radical formation was not observed in the dark-adapted state of the protein 
[30]. However, electron transfer and radical formation are observed in the light-adapted protein [39], most likely due to a tryptophan residue (W104, the only close tryptophan in

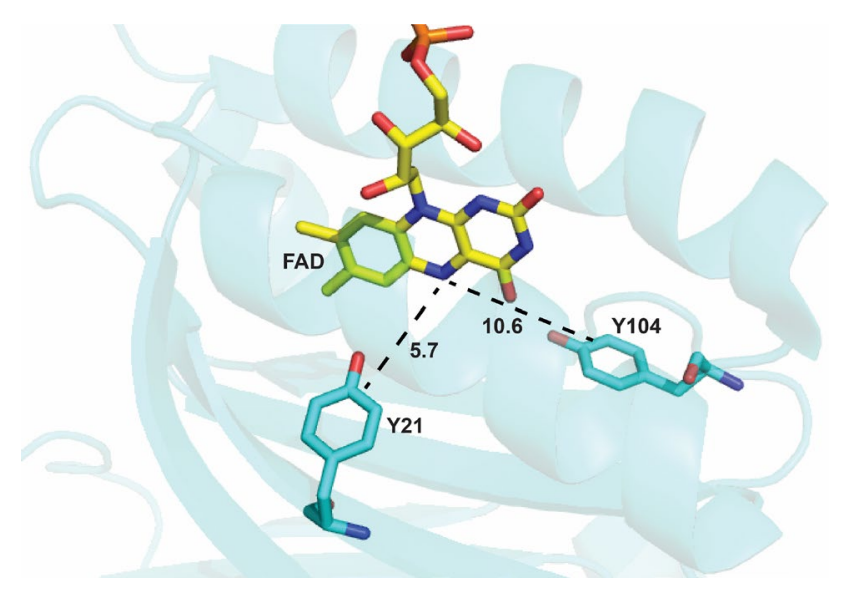

Fig. 5 Structure of AppA BLUF in light state (pdb: 2IYI) showing FAD and the two adjacent tyrosines. The position of Y104 is speculative, as the original structure contains a tryptophan at this position the protein) which moves closer to the flavin after blue-light absorption [40].

In this work, we replaced the close tryptophan (W104) with a tyrosine; therefore, Y21 and Y104 are the only two potential electron donors to flavin (see Fig. 5). We performed measurements on the light-adapted state of the W104Y mutant and our a priori expectation was that if we observe the build-up of an amino acid radical that must be a tyrosine radical.

Apart from the dominant excited state transients at 1380 and $1415 \mathrm{~cm}^{-1}$, the transient infrared spectra of the W104Y mutant (Fig. 6a) display the formation of transients at $\sim 1528 \mathrm{~cm}^{-1}$ (neutral flavin radical) as well as at $\sim 1502 \mathrm{~cm}^{-1}$. Both peaks were formed in approximately 2 ps as can be observed by the fits of these individual kinetics (Fig. 6c-d). Based on our findings in C51A TrmFO mutant and GOX, the $1502 \mathrm{~cm}^{-1}$ vibrational mode can be assigned to a neutral tyrosine radical and the presence of the $\sim 1528 \mathrm{~cm}^{-1}$ peak suggests that the W104Y mutant participates in proton-coupled electron transfer (PCET), thus forming $\mathrm{FADH}^{\circ}$ and $\mathrm{TyrO}^{\circ}$ to some extent.
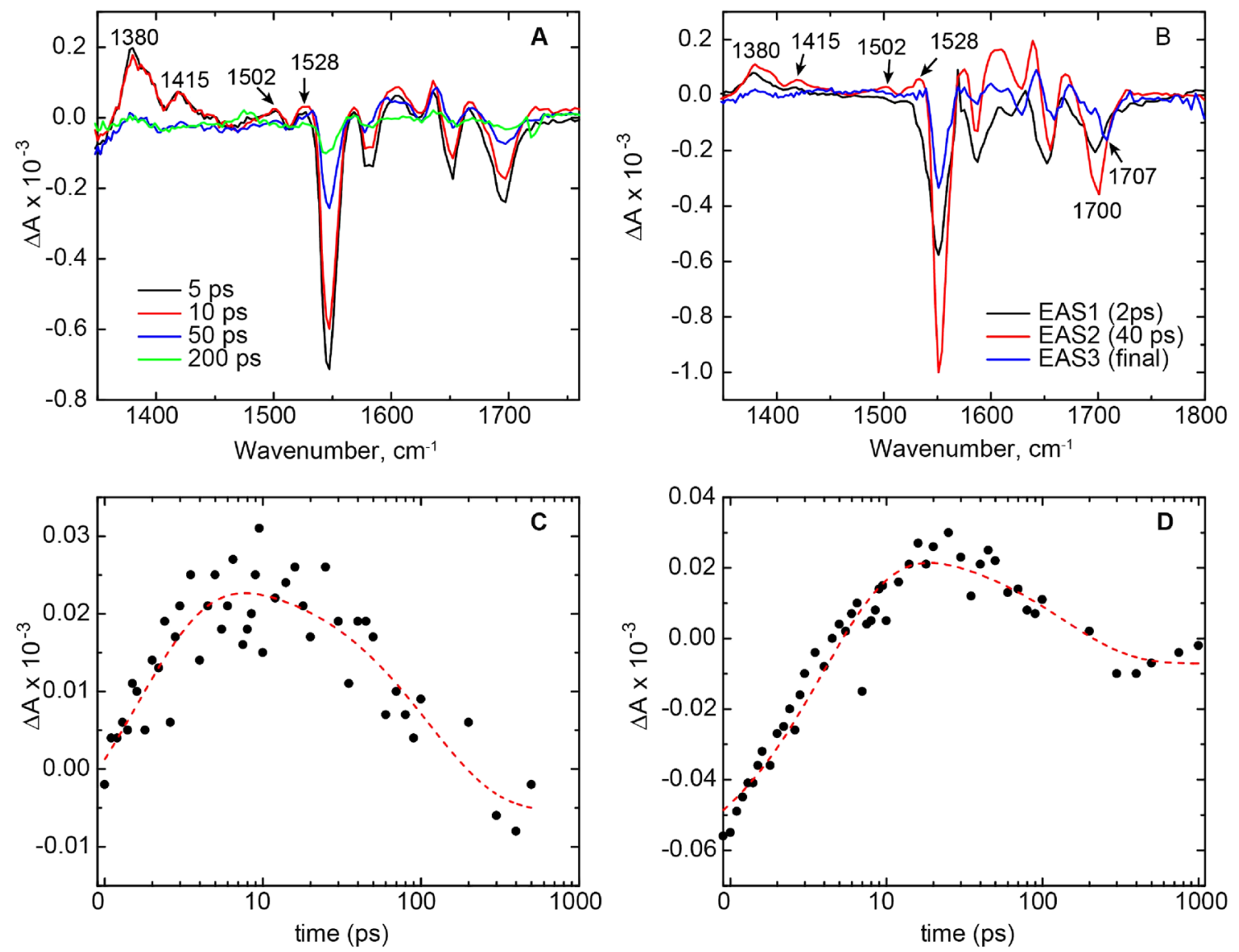

Fig. 6 a TRIR spectra measured on light-adapted state of W104Y AppABLUF at different delays. b EAS spectra from global analysis of the TRIR spectra measured on the W104Y AppA mutant. $\mathbf{c}$ Kinetics observed at $1502 \mathrm{~cm}^{-1}$. d Kinetics observed at $1528 \mathrm{~cm}^{-1}$ 
This assessment is corroborated by the global analysis (Fig. 6b). The EAS1 spectrum (2 ps) is dominated by vibrational modes of excited oxidized flavin. The EAS2 (40 ps) contains the appearance of both the formation of the neutral tyrosine radical as well as FADH. In the final EAS, the highfrequency bleach is shifted up $7 \mathrm{~cm}^{-1}$, reflecting that the $\mathrm{C} 4=\mathrm{O}$ of the flavin loses some hydrogen bond after the flavin becomes protonated. This result can be explained by the fact that tyrosine at 104 position is in hydrogen bonding distance to the flavin, and as it deprotonates the flavin loses that interaction, causing a substantial upshift of the $\mathrm{C} 4=\mathrm{O}$ stretch.

Indeed, we can conclude that activation of AppA W104Y mutant does not involve the sequential formation of charged radical species as observed in $\mathrm{C} 51 \mathrm{~A}$ TrmFO mutant and in GOX. Appearances of the neutral tyrosine radical and the neutral flavin radical peaks show evidence for a fraction of fast proton-coupled electron transfer process, in which tyrosine is the proton donor.

\section{Discussion}

We employed ultrafast infrared absorption measurements to identify the vibrational markers of the cation and neutral tyrosine radical in C51A and C51A/Y343F variants of TrmFO. We further investigated the formation of the same radical in wild type GOX and W104Y AppABLUF mutant in the light state. Comparing our previous visible transient absorption data measured on TrmFO mutants and GOX with the infrared measurements, we identify the $1483 \mathrm{~cm}^{-1}$ vibrational mode as a vibrational marker of $\mathrm{TyrOH}^{+}$. Tryptophan cation radical $\left(\mathrm{TrpH}^{+}\right)$has a vibrational signature around $1488-1490 \mathrm{~cm}^{-1}$. We were able to identify a vibrational marker for the neutral tyrosine radical at $1502 \mathrm{~cm}^{-1}$. The exact position of both the tyrosine cation and neutral radical strongly depends on the hydrogen bonding environment, on the solvent, so their frequencies can differ in a water-based buffer as in this work the samples were dissolved in deuterated buffer. The observed shift in a water-based buffer is expected to be smaller in the case of the neutral tyrosine radical and more significant in the case of the tyrosine cation radical.

This finding is in good agreement with earlier assignments of this vibrational mode with the pioneering work on phenoxyl and tyrosine radicals. Time-resolved Resonance Raman spectroscopy measurements-using $400 \mathrm{~nm}$ excitation-were done on phenoxyl radicals produced in aqueous solutions by pulse radiolysis. These measurements found a strong Raman peak at $1505 \mathrm{~cm}^{-1}$ which the authors assigned to the $\mathrm{C}=\mathrm{O}$ stretch of the phenol group [41]. A strong vibration for the tyrosine radical was found using UV Resonance Raman spectroscopy at $1510 \mathrm{~cm}^{-1}$ [20].

The role of tyrosine as electron donor in Photosystem II was first proved by Gerken, Brettel et al. using a nanosecond UV flash photolysis system; the authors proved that due to the electron transfer neutral tyrosine radical is formed [42]. Formation of neutral tyrosine radical in Photosystem II from Synechocystis sp. PCC 6803 was investigated by difference FTIR and the vibrational marker of $\operatorname{Tyr}_{\mathrm{D}}$ and $\mathrm{Tyr}_{\mathrm{Z}}$ radicals were found at 1503 and $1512 \mathrm{~cm}^{-1}$, respectively [17]. Calculations performed in the case of phenoxyl ( $\mathrm{PhO})$ and tyrosine radical (TyrO) revealed that the experimentally determined vibrational mode stands for the $\nu_{7 \mathrm{a}} \mathrm{C}-\mathrm{O}$ stretching of the phenol ring (see Table S1 and Fig. S4).

Using these new findings on the vibrational feature of neutral and cation tyrosine radical, we characterized the photochemistry of AppA W104Y mutant which was tailored to enhance and identify the formation of tyrosine radicals. This mutation altered significantly the photochemistry of the protein but as expected from earlier work [40, 43] it did not stop the formation of the light-adapted state, except it altered the dark-adapted state recovery to $\sim 100$-fold faster compared to WT AppA. Blue-light irradiation of BLUF domain proteins results in $\mathrm{a} \sim 10 \mathrm{~nm}$ shift of the $447 \mathrm{~nm}$ absorption peak of flavin due the reorganization of the hydrogen bonding network around the isoalloxazine ring. Stopping the irradiation, the absorption peak turns back to the original value in dark-adapted state. This recovery from light-adapted to dark-adapted state happens in 30 min in AppA and much faster (couple of seconds) in PixD or OaPAC. Replacement of W104 supresses the red shift in the full-length protein [43] and results in a faster recovery of the dark-adapted state in the BLUF domain; in W104A, the recovery was 80-fold faster than in wild type [44].

We performed transient infrared measurements on the light state of W104Y, and we observed one step formation of neutral tyrosine radical accompanying with a neutral flavin radical at $\sim 2$ ps after excitation. This suggests that in this mutant proton-coupled electron transfer occurs, with both the electron and proton donor being a tyrosine. PCET is not unexpected during the photoactivation of photoactive flavoproteins, especially if the primary electron donor is a tyrosine. Proton coupled electron transfer was observed for example in the case of two BLUF domain proteins, PixD [15] and PapB [45]. In the case of PixD, we observed a sequential radical formation: first, Y8 donated an electron to the flavin and FAD ${ }^{-}$radical formed, neutral flavin radical was formed concomitantly in $\sim 100$ ps [15]. Based on theoretical calculations, in the case of $\mathrm{PixD}$ protonation of the flavin happens sequentially: after excitation the tyrosine gives a proton to an adjacent glutamine which finally protonates the flavin, stabilizing the neutral radical state [46, 47]. In the case of PaPB, a BLUF protein found in the purple bacterium Rhodopseudomonas palustris, FADH was formed directly_as in the case of the AppA W104Y mutant_-via a proton-coupled electron transfer process [38]. 
Our experimental data indicate that exication of flavin in C51A TrmFO mutant also leads to a sequential protoncoupled electron transfer to a very small extent $(<10 \%)$, like in some of the BLUF domain proteins: tyrosine 343 gives an electron to the flavin forming the anionic flavin radical, subsequently fast deprotonation of the tyrosine leads to the protonation of the neutral flavin radical. We also have seen evidence for proton-coupled electron transfer in GOX, where an electron is transferred upon excitation from one of the close tyrosines, followed by a proton transferfrom the tyrosine which gave the electron-stabilizing the FADH state of flavin. Photochemistry of GOX was observed to be heterogenous and complex since besides the tyrosines, one of the close tryptophans can also donate an electron to the excited flavin. Altogether, this work exemplifies how extremely sensitive and selective TRIR method is in illucidating even minor reaction pathways involving aromatic residue radicals.

Supplementary Information The online version contains supplementary material available at https://doi.org/10.1007/s43630-021-00024-y.

Acknowledgements AL acknowledges funding from EFOP-3.6.216-2017-00005. A.L. and M.H.V. are grateful to the "Balaton" project (NKFIH 2017-2.2.5-TÉT-FR-2017-00005 and PHC Balaton 40173VE) for funding the travel exchanges. This study was supported by the National Science Foundation (NSF) (MCB-1817837 to PJT) and the EPSRC (EP/N033647/1 to S.R.M.). JNI was supported by a National Institutes of Health Chemistry-Biology Interface Training Grant (T32GM092714). JTC was supported by the IMSD-MERGE Program at Stony Brook University (5R25GM103962-04). The authors are grateful to STFC for access to the ULTRA laser facility.

Funding Open access funding provided by University of Pécs.

\section{Compliance with ethical standards}

Conflict of interest On behalf of all authors, the corresponding author states that there is no conflict of interest.

Open Access This article is licensed under a Creative Commons Attribution 4.0 International License, which permits use, sharing, adaptation, distribution and reproduction in any medium or format, as long as you give appropriate credit to the original author(s) and the source, provide a link to the Creative Commons licence, and indicate if changes were made. The images or other third party material in this article are included in the article's Creative Commons licence, unless indicated otherwise in a credit line to the material. If material is not included in the article's Creative Commons licence and your intended use is not permitted by statutory regulation or exceeds the permitted use, you will need to obtain permission directly from the copyright holder. To view a copy of this licence, visit http://creativecommons.org/licenses/by/4.0/.

\section{References}

1. Byrdin, M., Villette, S., Espagne, A., Eker, A., \& Brettel, K. (2008). Polarized transient absorption to resolve electron transfer between tryptophans in DNA photolyase. Journal of Physical Chemistry B, 112(22), 6866-6871.

2. Aubert, C., Vos, M. H., Mathis, P., Eker, A. P., \& Brettel, K. (2000). Intraprotein radical transfer during photoactivation of DNA photolyase. Nature, 405(6786), 586-590.

3. Lukacs, A., Eker, A., Byrdin, M., Brettel, K., \& Vos, M. (2008). Electron hopping through the 15 angstrom triple tryptophan molecular wire in DNA photolyase occurs within $30 \mathrm{ps.} \mathrm{Journal}$ of the American Chemical Society, 130(44), 14394-14395.

4. Liu, Z., Zhang, M., Guo, X., Tan, C., Li, J., Wang, L., et al. (2013). Dynamic determination of the functional state in photolyase and the implication for cryptochrome. Proceedings of the National Academy of Sciences of the United States of America, 110(32), 12972-12977.

5. Liu, Z., Tan, C., Guo, X., Li, J., Wang, L., Sancar, A., \& Zhong, D. (2013). Determining complete electron flow in the cofactor photoreduction of oxidized photolyase. Proceedings of the National Academy of Sciences of the United States of America, 110(32), 12966-12971.

6. Brazard, J., Usman, A., Lacombat, F., Ley, C., Martin, M. M., Plaza, P., et al. (2010). Spectro-temporal characterization of the photoactivation mechanism of two new oxidized cryptochrome/ photolyase photoreceptors. Journal of the American Chemical Society, 132(13), 4935-4945.

7. Cailliez, F., Müller, P., Gallois, M., \& de la Lande, A. (2014). ATP binding and aspartate protonation enhance photoinduced electron transfer in plant cryptochrome. Journal of the American Chemical Society, 136(37), 12974-12986.

8. Müller, P., Bouly, J. P., Hitomi, K., Balland, V., Getzoff, E. D., Ritz, T., \& Brettel, K. (2014). ATP binding turns plant cryptochrome into an efficient natural photoswitch. Scientific Reports, 4, 5175.

9. Müller, P., Brettel, K., Grama, L., Nyitrai, M., \& Lukacs, A. (2016). Photochemistry of wild-type and N378D mutant E. coli DNA photolyase with oxidized FAD cofactor studied by transient absorption spectroscopy. ChemPhysChem, 17(9), 1329-1340.

10. Müller, P., \& Bouly, J. P. (2015). Searching for the mechanism of signalling by plant photoreceptor cryptochrome. FEBS Letters, 589(2), 189-192.

11. Kötting, C., \& Gerwert, K. (2005). Proteins in action monitored by time-resolved FTIR spectroscopy. ChemPhysChem, 6(5), 881-888.

12. Wolf, M. M., Schumann, C., Gross, R., Domratcheva, T., \& Diller, R. (2008). Ultrafast infrared spectroscopy of riboflavin: Dynamics, electronic structure, and vibrational mode analysis. The Journal of Physical Chemistry B, 112(42), 13424-13432.

13. Nibbering, E. T., Fidder, H., \& Pines, E. (2005). Ultrafast chemistry: Using time-resolved vibrational spectroscopy for interrogation of structural dynamics. Annual Review of Physical Chemistry, 56, 337-367.

14. Zhu, J., Mathes, T., Stahl, A. D., Kennis, J. T., \& Groot, M. L. (2012). Ultrafast mid-infrared spectroscopy by chirped pulse upconversion in 1800-1000 $\mathrm{cm}(-1)$ region. Optics Express, 20(10), 10562-10571.

15. Gil, A. A., Laptenok, S. P., Iuliano, J. N., Lukacs, A., Verma, A., Hall, C. R., et al. (2017). Photoactivation of the BLUF protein PixD probed by the site-specific incorporation of fluorotyrosine residues. Journal of the American Chemical Society, 139(41), 14638-14648.

16. Dixon, W., \& Murphy, D. (1976). Determination of acidity constants of some phenol radical cations by means of electron-spin resonance. Journal of the Chemical Society-Faraday Transactions II, 72, 1221-1230.

17. Berthomieu, C., \& Hienerwadel, R. (2005). Vibrational spectroscopy to study the properties of redox-active tyrosines in 
photosystem II and other proteins. BiochimicaetBiophysica Acta, 1707(1), 51-66.

18. Tomter, A. B., Zoppellaro, G., Bell, C. B., Barra, A. L., Andersen, N. H., Solomon, E. I., \& Andersson, K. K. (2012). Spectroscopic studies of the iron and manganese reconstituted tyrosyl radical in Bacillus cereus ribonucleotide reductase R2 protein. PLOS ONE, 7(3), e33436.

19. Solar, S., Gtoff, N., Surdhar, P. S., Armstrong, D. A., \& Singh, A. (1991). Oxidation of tryptophan and $\mathrm{N}$-methylindole by $\mathrm{N} 3, \mathrm{Br}_{2}{ }_{2}$ and $(\mathrm{SCN})_{2}{ }^{--}$radicals in light- and heavy-water solutions: A pulse radiolysis study. Journal of Physical Chemistry, 95, 3639-3643.

20. Johnson, C., Ludwig, M., \& Asher, S. (1986). Ultraviolet resonance Raman characterization of photochemical transients of phenol, tyrosine, and tryptophan. Journal of the American Chemical Society, 108(5), 905-912.

21. Nag, L., Sournia, P., Myllykallio, H., Liebl, U., \& Vos, M. (2017). Identification of the $\mathrm{TyrOH}(\cdot+)$ radical cation in the flavoenzyme TrmFO. Journal of the American Chemical Society, 139(33), 11500-11505.

22. Nag, L., Lukacs, A., \& Vos, M. H. (2019). Short-lived radical intermediates in the photochemistry of glucose oxidase. ChemPhysChem, 20(14), 1793-1798.

23. Nishimasu, H., Ishitani, R., Yamashita, K., Iwashita, C., Hirata, A., Hori, H., \& Nureki, O. (2009). Atomic structure of a folate/ FAD-dependent tRNAT54 methyltransferase. Proceedings of the National Academy of Sciences of the United States of America, 106(20), 8180-8185.

24. Iuliano, J. N., Gil, A. A., Laptenok, S. P., Hall, C. R., Tolentino Collado, J., Lukacs, A., et al. (2018). Variation in LOV photoreceptor activation dynamics probed by time-resolved infrared spectroscopy. Biochemistry, 57(5), 620-630.

25. Greetham, G., Burgos, P., Cao, Q., Clark, I., Codd, P., Farrow, R., et al. (2010). ULTRA: A unique instrument for time-resolved spectroscopy. Applied Spectroscopy, 64(12), 1311-1319.

26. Brust, R., Lukacs, A., Haigney, A., Addison, K., Gil, A., Towrie, M., et al. (2013). Proteins in action: Femtosecond to millisecond structural dynamics of a photoactive flavoprotein. Journal of the American Chemical Society, 135(43), 16168-16174.

27. van Stokkum, I. H., Larsen, D. S., \& van Grondelle, R. (2004). Global and target analysis of time-resolved spectra. Biochimica et Biophysica Acta, 1657(2-3), 82-104.

28. Haigney, A., Lukacs, A., Zhao, R. K., Stelling, A. L., Brust, R., Kim, R. R., et al. (2011). Ultrafast infrared spectroscopy of an isotope-labeledphotoactivatable flavoprotein. Biochemistry, 50(8), 1321-1328.

29. Haigney, A., Lukacs, A., Brust, R., Zhao, R. K., Towrie, M., Greetham, G. M., et al. (2012). Vibrational assignment of the ultrafast infrared spectrum of the photoactivatable flavoprotein AppA. The Journal of Physical Chemistry B, 116(35), 10722-10729.

30. Lukacs, A., Brust, R., Haigney, A., Laptenok, S. P., Addison, K., Gil, A., et al. (2014). BLUF domain function does not require a metastable radical intermediate state. Journal of the American Chemical Society, 136(12), 4605-4615.

31. Lukacs, A., Zhao, R. K., Haigney, A., Brust, R., Greetham, G. M., Towrie, M., et al. (2012). Excited state structure and dynamics of the neutral and anionic flavin radical revealed by ultrafast transient mid-IR to visible spectroscopy. The Journal of Physical Chemistry B, 116(20), 5810-5818.

32. Dozova, N., Lacombat, F., Bou-Nader, C., Hamdane, D., \& Plaza, P. (2019). Ultrafast photoinduced flavin dynamics in the unusual active site of the tRNA methyltransferase TrmFO. Physical Chemistry Chemical Physics, 21(17), 8743-8756.

33. Walden, S., \& Wheeler, R. (1996). Distinguishing features of indolyl radical and radical cation: Implications for tryptophan radical studies. Journal of Physical Chemistry, 100(5), 1530-1535.

34 Walden, S., \& Wheeler, R. (1996). Structural and vibrational analysis of indole by density functional and hybrid Hartree-Fock/density functional methods. Journal of the Chemical Society-Perkin Transactions 2, 12, 2653-2662.

35. Shafaat, H., \& Kim, J. (2014). Resonance Raman analysis of the tryptophan cation radical. Journal of Physical Chemistry Letters, 5(17), 3009-3014.

36. Blanco-Rodriguez, A., Towrie, M., Sykora, J., Zalis, S., \& Vlcek, A. (2011). Photoinduced intramolecular tryptophan oxidation and excited-state behavior of $[\operatorname{Re}(\mathrm{L}-\mathrm{AA})(\mathrm{CO})(3)($ alpha-diimine $)](+)$ $(\mathrm{L}=$ pyridine or imidazole, $\mathrm{AA}=$ tryptophan, tyrosine, phenylalanine). Inorganic Chemistry, 50(13), 6122-6134.

37. Masuda, S. (2013). Light detection and signal transduction in the BLUF photoreceptors. Plant and Cell Physiology, 54(2), 171-179.

38. Masuda, S., Hasegawa, K., \& Ono, T. A. (2005). Light-induced structural changes of apoprotein and chromophore in the sensor of blue light using FAD (BLUF) domain of AppA for a signaling state. Biochemistry, 44(4), 1215-1224.

39. Toh, K., van Stokkum, I., Hendriks, J., Alexandre, M., Arents, J., Perez, M., et al. (2008). On the signaling mechanism and the absence of photoreversibility in the AppABLUF domain. Biophysical Journal, 95(1), 312-321.

40. Karadi, K., Kapetanaki, S. M., Raics, K., Pecsi, I., Kapronczai, R., Fekete, Z., et al. (2020). Functional dynamics of a single tryptophan residue in a BLUF protein revealed by fluorescence spectroscopy. Sci Rep, 10(1), 2061.

41. Tripathi, G., \& Schuler, R. (1984). The resonance Raman-spectrum of phenoxyl radical. Journal of Chemical Physics, 81(1), 113-121.

42. Gerken, S., Brettel, K., Schlodder, E., \& Witt, H. (1988). Optical characterization of the immediate electron-donor to chlorophyllAII+ IN O-2-evolving photosystem-II complexes-tyrosine as possible electron carrier between chlorophyll-AII and the wateroxidizing manganese complex. FEBS Letters, 237(1-2), 69-75.

43. Dragnea, V., Arunkumar, A., Yuan, H., Giedroc, D., \& Bauer, C. (2009). Spectroscopic studies of the AppABLUF domain from Rhodobacter sphaeroides: Addressing movement of tryptophan 104 in the signaling state. Biochemistry, 48(42), 9969-9979.

44. Masuda, S., Tomida, Y., Ohta, H., \& Takamiya, K. (2007). The critical role of a hydrogen bond between Gln63 and Trp104 in the blue-light sensing BLUF domain that controls AppA activity. Journal of Molecular Biology, 368(5), 1223-1230.

45. Fujisawa, T., Takeuchi, S., Masuda, S., \& Tahara, T. (2014). Signaling-state formation mechanism of a BLUF protein PapB from the purple bacterium Rhodopseudomonas palustris studied by femtosecond time-resolved absorption spectroscopy. The Journal of Physical Chemistry B, 118(51), 14761-14773.

46. Goings, J., \& Hammes-Schiffer, S. (2019). Early photocycle of Slr1694 blue-light using flavin photoreceptor unraveled through adiabatic excited-State quantum mechanical/molecular mechanical dynamics. Journal of the American Chemical Society, 141(51), 20470-20479.

47. Goings, J., Reinhardt, C., \& Hammes-Schiffer, S. (2018). Propensity for proton relay and electrostatic impact of protein reorganization in Slr1694BLUF photoreceptor. Journal of the American Chemical Society, 140(45), 15241-15251. 